


\section{Pedagogies of Mapping}

\section{Rebecca Johnson*}

Generations of students have engaged in the (more or less artistic) practice of doodling in the margins of their notes, and yet it is rare for law students to be given crayons and be directed to colour. In this note, I describe and reflect on the experience of using the visually based pedagogy of "mapping" as a tool for exploring the Insite case. This exercise took place at the end of the Legal Process module, after students had spent nearly two days of concentrated attention on the case and the issues raised by it. The class was divided into four groups, each of which was asked to imagine themselves as a newly formed government working group charged with the task of imagining more visionary ways of dealing with the problems of the "hard to house, hard to reach and hard to treat." The first task was to work as a group to map out the terrain on which new solutions might be developed: to depict visually the hopes, fears, concerns, difficulties, convergences and possible strategic alliances created by drug use in the Downtown East Side (DTES). ${ }^{1}$

While some of the students expressed worry about their lack of drawing skills, the group took up the challenge with good humour. They were given coloured pens, large sheets of paper, and roughly 45 minutes to talk and draw. The groups then returned with their maps, each of which is reproduced below. ${ }^{2}$ We hung them up in the classroom, and heard from each group about what they had tried to capture in their map, as well as the difficulties and challenges they faced in deciding what they would capture. Here, they talked about some of the pragmatic limits to their maps (such as whether or not groups had members with adequate skills in drawing), as well as about some of the more conceptual challenges involved in finding ways to represent complex social and legal concepts visually. This included questions about the role of "time" or "place" in determining what a map might look like, as well as the implications of focusing visual attention on different dimensions of the problem. The exercise also generated a lively discussion about the dynamics of group decision-making processes, and the relationship of those processes to their efforts to produce some kind of "common" mapping.

One of the most interesting dimensions of the exercise arose from looking at the four maps in conversation with each other. Indeed, each of the maps seemed to capture different metaphors for the Insite case, metaphors that undoubtedly circulate in a number of complicated social/legal contexts. In brief, one could say that participants visualized the case in four different ways: one focusing on people, another on place, a third on fabric, and a fourth on flows of power. These different visual metaphors are visible as we examine each map in turn. First, consider the map that focused on "the people" (see page 140).

This map was generated by the group that inherited one of the "artists" in the class. The group drew on this expertise in art to produce a mapping that involved a playful echo between Da Vinci's painting "The Last Supper" ${ }^{3}$ and the various people the students had encountered over the week (in the panel of experts who came to speak, and the people featured in Nettie Wild's film, Fix). Vancouver Mayor Phillip Owen sits at the centre of the table, framed by various advocates for and against Insite. On the left are activist Ann Livingston and addict Dean Wilson (both featured in the movie, Fix). A hypodermic needle is visible in Dean's hand. On the right sit a number of advocates from the business community (again, featured 
MAP 1: People.... “The Last Supper"

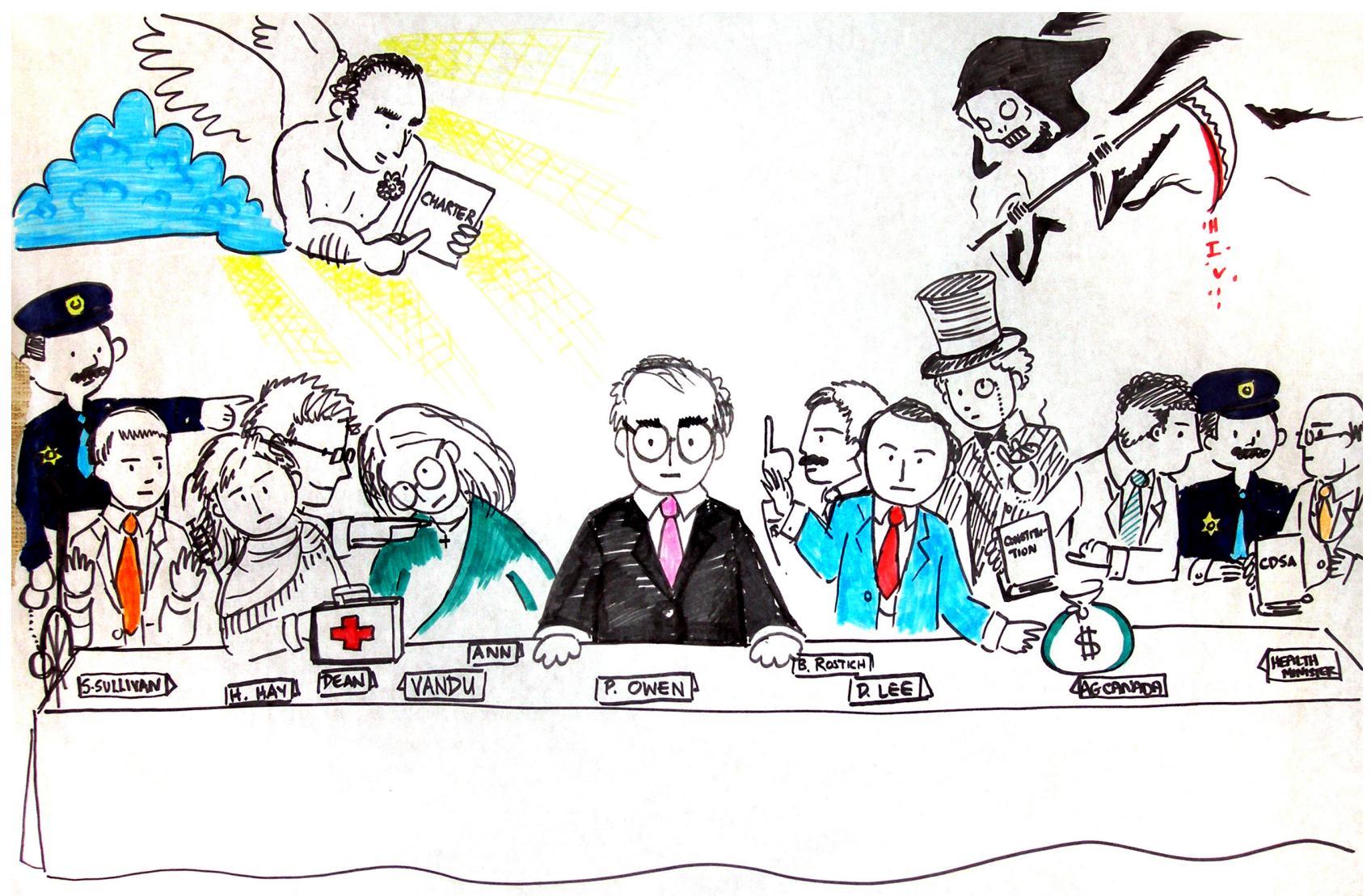

in the film Fix). Note that the Health Authority and Minister of Health are on opposite sides of the table. Note also, however, that the Police are found on both sides of the table, spatialized as representing communities on both the left and the right of any issue. The "monopoly man" on the left (nicknamed "Mr. Kerrisdale" by the group), evokes the ongoing discussions that had occurred in class about the differences between residents of the DTES and of the more upscale Vancouver neighbourhoods. In the top corners, an angelic Pierre Trudeau holds out the Charter, while Death wields a scythe, from which drip the letters, "H.I.V."

This map places all the players at a table together, locating them spatially in specific relationships to each other (i.e., people on the left, people on the right, people in the middle, police officers on both sides, etc.). This map also captures something of the processes involved in group decision-making. Their placement at a table captures the way in which different forms of decision-making require people to interact with each other and discuss problems in concrete locations. Situating the mayor at the centre also tended to foreground the questions of politics and democracy circling around the case.

Group Two took a different approach. This group took up the challenge of mapping in a literal way, attending to the importance of place. Here, using the hypodermic needle as a marker, the group placed Insite in its accurate geographical location in the DTES. Across the street, the circle references the Carnegie Community Centre as a reminder that the downtown is not just a place, but a community. Both the Courts (represented by the scales of justice) and Police Department (represented by handcuffs) are also visible in the area as institutions involved in the life of the downtown. The overlapping bubbles of 
MAP 2: Places ... “Downtown”

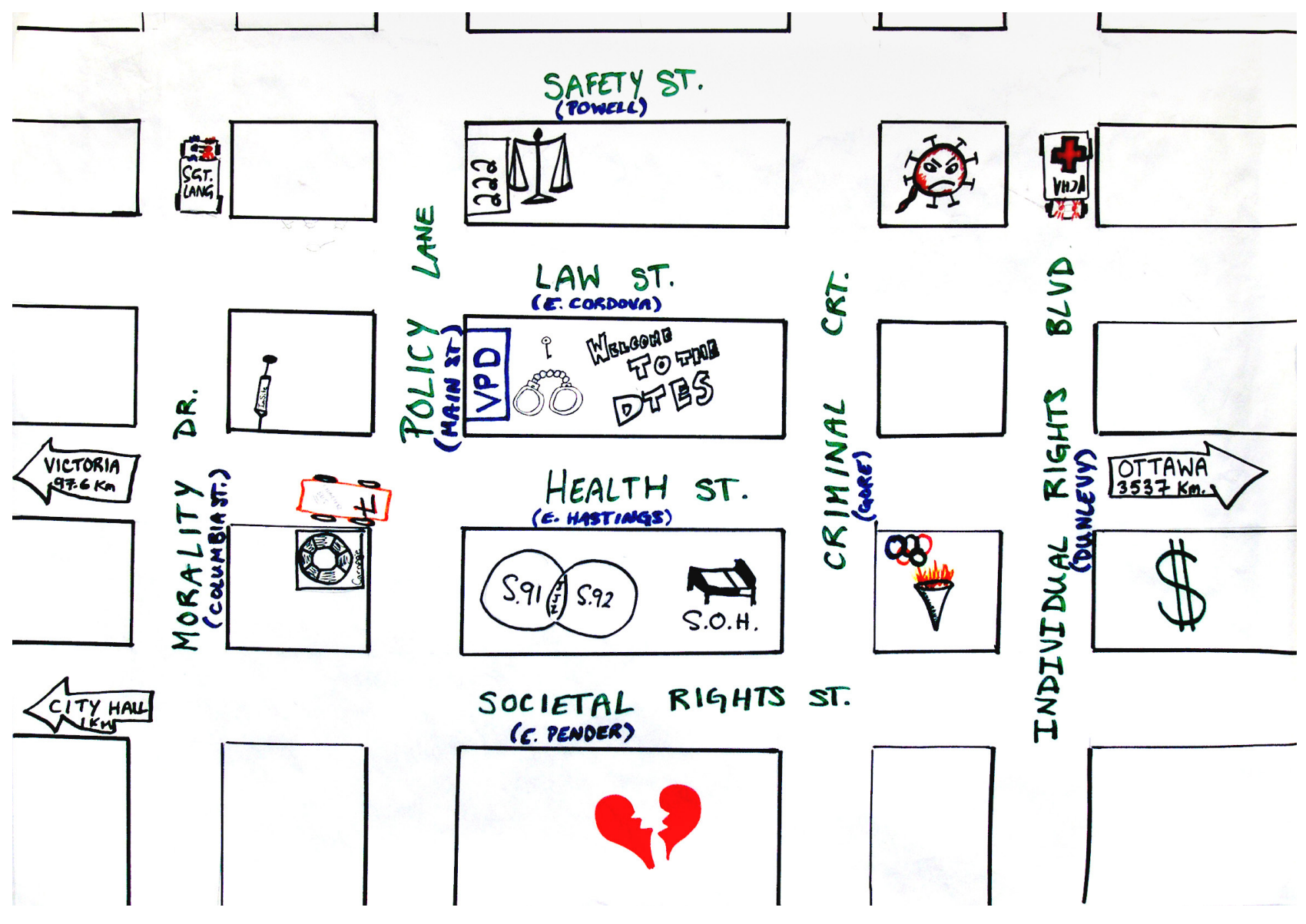

section 91 and section 92 jurisdiction make visible the ways Constitutional debates play themselves out in particular places. The streets on this map carry both their real names (Pender, Hastings, Cordova), along with names marking out the issues Insite raises for the downtown community (Safety, Health, Morality, etc). On the streets themselves, Sgt Lang (featured in the movie Fix) patrols the beat in his police car, while both an ambulance, and the Section 7 Charter bus roll up and down the streets. This mapping captures additional debates about place that were circling around the case: the bed and initials S.O.H. reference the difficulties of the "singleoccupant-housing" situation featured in the documentary Staying Alive. The Olympic torch and logo are reminders of attempts to clean up the area for the Winter Games. At the bottom of the map, there is an evocation of "the street of broken dreams." On the edges of the map, there is a further statement about the politics of the local, with signage markers indicating how far one must go from the DTES to get to politicians at the municipal, provincial and federal levels. The map leaves it to the viewer to decide if the differences here relate to being "west and east" or "left and right" in a political sense.

Group 3 produced a map that could be best described using the metaphor of fabric or of a web. Whether constructed by spider or loom, the metaphor points in the direction of something woven from threads. It suggests a fabric with warp and woof threads that pull on each other and are in tension with each other. Like the edges of a loom, Health, Power, Order, and Economy seem to provide points of stability, but one can see that those seeming points of stability have more flexibility in them than one might expect. Not all economic arguments, for example, pull in the same direction. Economic argu- 
MAP 3: Fabric: Weaving a Web

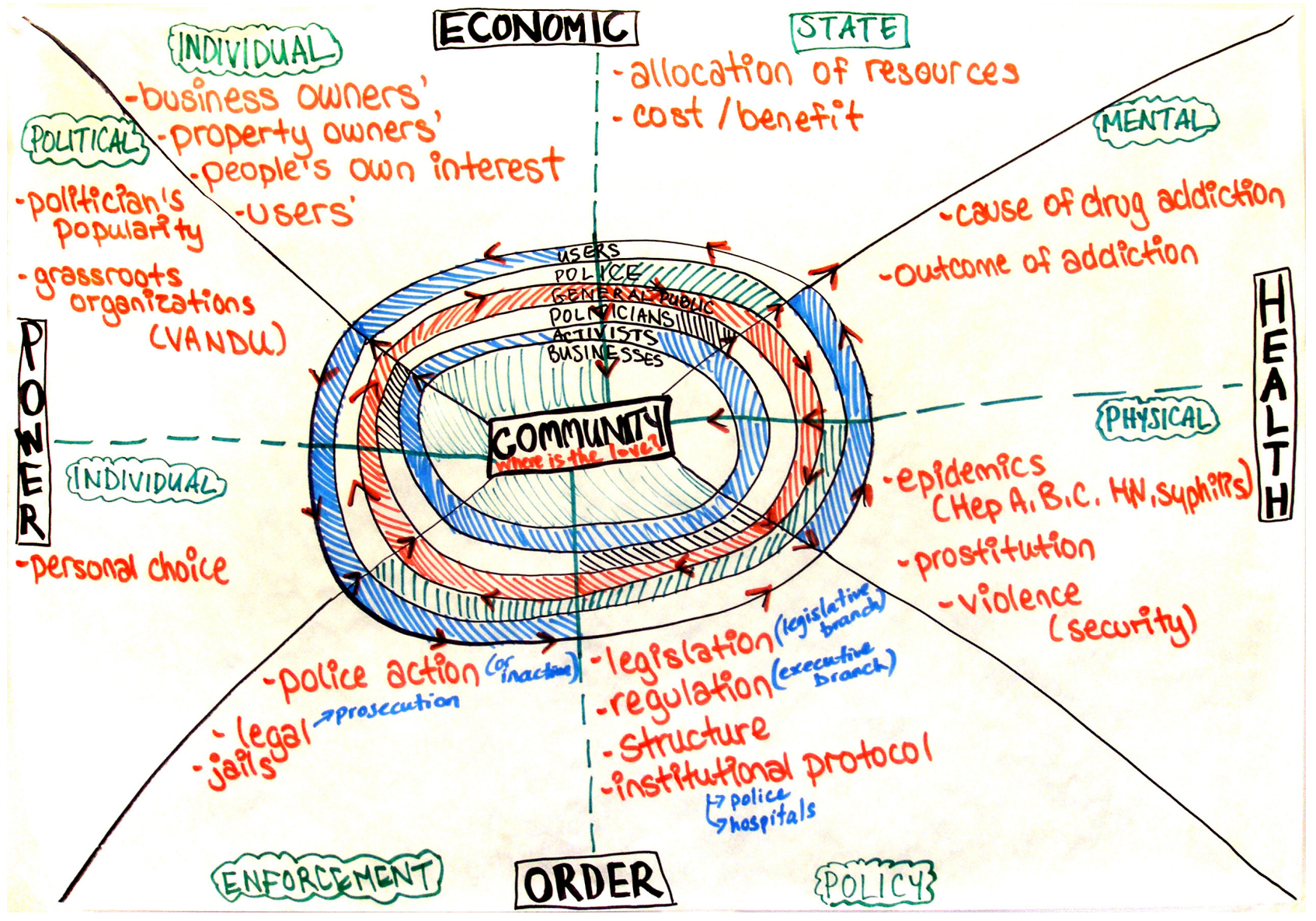

ments were constructed both for and against Insite. Health arguments were made both for and against Insite. Arguments about Order were similarly constructed in ways that left space for reasonable disagreement about what policy or approach would best support social order.

Woven into the lines that traverse the case are a series of concentric circles with the word "community" at the centre. But the circles making up that community include distinct circles for business, activists, politicians, users, police, and the general public. Directions of pull are indicated in these smaller circles, in ways that also make visible the differences, for example, between individual and state interests that various communities might feel (or not feel) in at different locations on the fabric. This map makes visible the ways that no group of people touched by the Insite case was monolithic in its interests or understandings of the case. And indeed, this mapping reminds us that the communities affected by the case were not always discrete and separate. In a mapping such as this, one captures a sense of the ways in which issues were woven together, and the ways in which a tug on a single thread could reverberate throughout the fabric.

The fourth group produced a map with two layers of paper. On the top layer, they attempted to map out some of the processes and flows they had seen over the week. While one might say this is in some ways the messiest map, it is also one that provides the clearest sense of relations between, for example, health, economy, poverty, government, economy and family. Communities and groupings of people are situated around the edges of the map, with arrows running through the centre, raising questions about causation, correlation, and connection. This map also points to questions of time and 
Map 4:Flows . . . Overlapping Layers

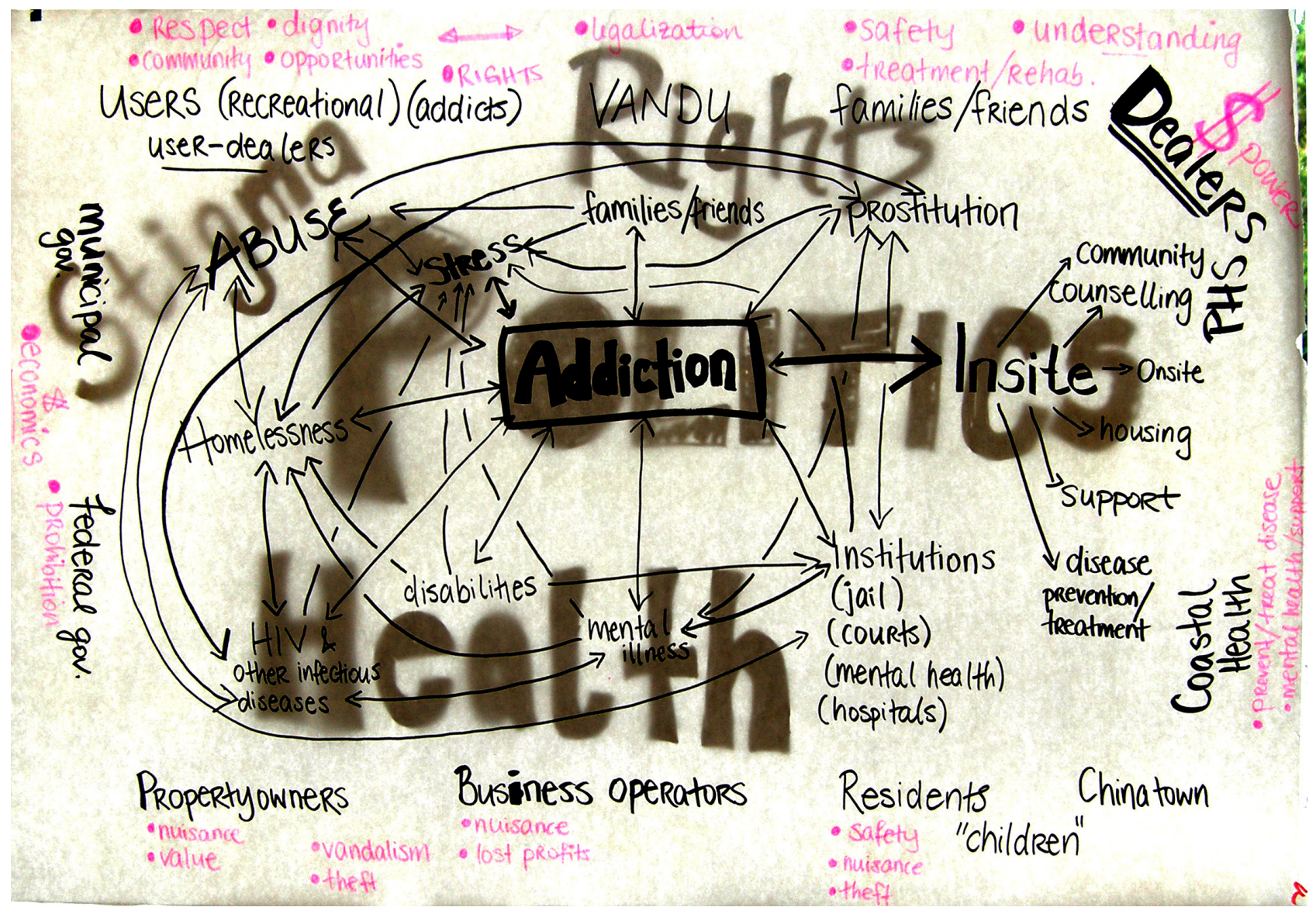

movement, in contrast to the relative fixity seen in the other maps. The second layer of the map contains a series of concepts (or interests): Health, Politics, Rights, and Stigma. These are concepts that exerted powerful pressures on the debates and decision-making processes in the forming of Insite. They were not initially visible when the map was hung up on the board. It was only when we hung the map up on the window that the words showed through as ghostly underimages. The map was a reminder of the ways different assumptions and presumptions about these concepts are continually bleeding through into the conversations from below in ways that are not always visible, but that nonetheless inflect the present, and our understandings of the flows and movements of power and possibility.

\section{Final comments}

The maps produced by the groups were independently interesting. But the most useful part of the exercise was the debrief session at the end of our class, and the chance it gave us to consider how each of the four different maps provides another lens for understanding the case and its challenges. The exercise did not lead to the conclusion that any particular mapping was the right way to capture the case (nor even that mapping is always a productive tool!). But in this particular context, we saw that each map provided another interesting vehicle for thinking through the ways in which one might understand the various aspects of the case. In this context, the different maps enabled us to focus on people, on places, on institutions, on weaving, and on process and flow. Having the four lenses gave us a broader range of tools for un- 
derstanding and for sharing our observations. It made visible particular challenges and tools; how different ways of mapping demand the erasure or neglect of some dimensions of experience; and about the perennial difficulties lawyers, judges and litigants face in translating our understandings of law and justice from the medium of experience to that of images or words, and back again.

\section{Notes}

* Professor, Faculty of Law, University of Victoria.

1 The instructions given to the students for the mapping exercise can be found on the web at http://insite.law.uvic.ca.

2 Here, creative rights in the maps are undoubtedly held by the fabulous students in that section of legal process, students who cast themselves into the task (or, who were cast by us into the task) with wholehearted (and a measure of justifiably faint-hearted) abandon. The authors in this context were: Asif Abdulla, Stephanie AshleyPryce, Jean-Kyle Bienvenu, Trina Brubaker, Geoff Coombs, Rebecca Crookshanks, Michael Gismondi, Natasha Gooch, Rory Johnston, Lianne Kramchynski, Agnes Lee, Ainsley MacCallum, Jeff Miller, Miles Motture, Laura Nichols, Brian Smith, Devon Peck, Dana Phillips, Greg Piper, Julia Tchezganova, Heather Watt, Michael Weber. The colour versions of the photos can be seen at http://insite.law.uvic.ca. Photos of the maps were taken (and cleaned up!) by Thomas Winterhoff, Communications Officer, University of Victoria Faculty of Law.

3 http://milan.arounder.com/en/churches/santamaria-delle-grazie-church/the-last-supper-leonardo-da-vinci.html. 